\title{
Viscous Fluid Flowing around the Plate: Turbulence
}

\author{
Alexander Ivanchin \\ Orpheus Ltd., Tomsk, Russia \\ Email: al.g.ivanchin@gmail.com
}

How to cite this paper: Ivanchin, A. (2020) Viscous Fluid Flowing around the Plate: Turbulence. Open Journal of Fluid Dynamics, 10, 291-316.

https://doi.org/10.4236/ojfd.2020.104018

Received: September 16, 2020

Accepted: November 23, 2020

Published: November 26, 2020

Copyright $\odot 2020$ by author(s) and Scientific Research Publishing Inc. This work is licensed under the Creative Commons Attribution International License (CC BY 4.0).

http://creativecommons.org/licenses/by/4.0/

\begin{abstract}
Diffusion of momentum gives rise to viscosity. This article presents a solution in the explicit form of the equation of the momentum diffusion for a viscous fluid flowing around a plate taking into account deceleration. Three characteristic regions of a viscous flow have been described: the mantle, the body of the boundary layer, the viscous sublayer. In the mantle, the effect of viscosity is significant at a considerable distance from the plate. The momentum diffusion is focused in the body of the boundary layer. The diffusion force that produces the momentum of force giving rise to eddies is localized in the viscous sublayer. At the beginning of the plate, a moment of force twists the liquid along the flow, creating eddies that roll along the plate. For this reason, they are pressed against the surface of the plate. But at some distance from the beginning of the plate, the moment of force changes its orientation to the opposite and twists the vortices in the opposite direction, causing the vortices to roll along the plate against the flow. This causes the liquid to detach from the surface of the plate. This is the beginning of turbulence. The diameter of the vortex produced in the viscous sublayer is small being of the order of the thickness of the viscous sublayer. The vortex possesses a large angular velocity. Due to the momentum diffusion and the effect of the eddies combined in passing along the plate, its diameter increases up to the size of the thickness of the boundary layer and even more, whereas its angular velocity decreases down to the values really observed. The value of the critical Reynolds number of the transition from the laminar flow to the turbulent one has been found, and it agrees with the experimental data. The value of the shear stress produced by the viscous fluid on the plate surface has also been obtained. The way of measurement of the friction coefficient characterizing the effect of the plate on the flow has been proposed. It has been shown that the boundary condition of adhesion to the surface of a body flown around, that is applied in the estimation of viscous flows, contradicts the real processes of the flow.
\end{abstract}




\section{Keywords}

Viscous Fluid Mechanics, Momentum Diffusion, Viscous Flow around the Plate, Generation of Vortices, Reynolds Critical Number

\section{Introduction}

At present, the theory of the flow of a viscous fluid has been insufficiently developed. Even the simplest problem of a flow around a plate parallel to the stream cannot be solved using the proposed solutions that are not suitable for the description of the flow near the front edge of the plate. Nor there is an adequate description of the transition of the laminar flow to the turbulent one or the theory of the generation of eddies, etc. The main reason for this situation is the introduction of "viscous stresses" into the above theory, that do not exist in nature [1]. At the same time, it is surprising that when considering a viscous flow, they are absolutely right in saying that the reason for the viscosity is the momentum diffusion. However, instead of studying diffusion, they say that it produces the shear stresses $\tau$.

The Newton law is postulated

$$
\tau=\eta \frac{\partial u_{x}}{\partial y}
$$

here $u_{x}$ is the velocity of the flow along the abscissa; $\eta$ is the viscosity coefficient. By definition, a fluid and a gas are of the media in which there are no shear stresses. The momentum is a volume vector. The forces produced by its diffusion are also volume forces rather than surface once, the latter being characteristic of a shear stress. An incorrect definition of this force is the reason for misunderstanding of a viscous flow. As a result, the fictitous viscous stresses have been included in the equation of motion, which made it unsuitable for calculations. Thus, the diffusion equation, which is the key element in the theory of viscosity, has escaped consideration.

\section{Equation of Momentum Diffusion}

If a fluid contains the momentum sources with the volume density $\boldsymbol{J}$, then, according to the Newton law [2] [3] [4] [5]

$$
\frac{\mathrm{d} \boldsymbol{J}}{\mathrm{d} t}=\boldsymbol{F}
$$

The momentum sources are equivalent to the action of the volume force. Here $\boldsymbol{J}, \boldsymbol{F}$ are the vectors of the momentum density and the volume force density, respectively. The consideration is carried out under the two-dimensional statement of the problem, with the problem parameters depending only on two space variables $x$ and $y$. Figure 1 shows a schematic of the considered problem. The stationary plate of the length $\mathrm{d} l$ flown around by a viscous fluid is located on 

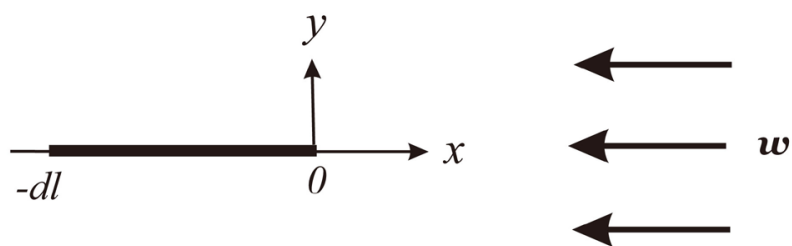

Figure 1. Shown is a plate of the length $\mathrm{d} l$, located on the abscissa and flown around by a viscous fluid with the momentum density $\boldsymbol{w}$.

the abscissa interval $(-\mathrm{d} l, 0)$. The flow of the viscous fluid with the momentum density

$$
\boldsymbol{w}=\{-w, 0\}=\left\{-v_{e} \rho, 0\right\}
$$

is parallel to the abscissa in the negative direction. Here $v_{e}>0$ is the velocity of the approach stream, $\rho$ is the density. The minus in (3) means that the flow moves in the negative direction of the abscissa. The diffusion vector of the momentum $\boldsymbol{I}=\{I, 0\}$ also consists of one abscissa component. The viscosity is produced by the momentum diffusion [1]. The effect of the momentum produced by diffusion can be treated as the appearance of the momentum sources with the density $\boldsymbol{J}=\{J, 0\}$ in the flow that are derived from the equation of the momentum diffusion.

\subsection{Diffusion Equation}

Dimensionless equation. The nonstationary equation of momentum diffusion in a motionless fluid is

$$
\frac{\partial J}{\partial t}-\eta\left(\frac{\partial^{2} J}{\partial x^{2}}+\frac{\partial^{2} J}{\partial y^{2}}\right)=J_{0} \mathrm{~d} l \mathrm{~d} t \delta(x) \delta(y) \delta(t)
$$

Here $\eta$ is the diffusion coefficient, $J_{0}$ is the power of the momentum source on the plate, i.e. the momentum quantity produced by unit of surface per unit of time, $\mathrm{d} l$ is the length of the elementary plate flown around. The value of $J_{0} \mathrm{~d} l \mathrm{~d} t$ is the quantity of the momentum produced when the momentum source operates on the plate of the length $\mathrm{d} l$ during the time $\mathrm{d} \tau$. The length $\mathrm{d} l$ is considered small.

Equation (4) is rewritten as

$$
\frac{\partial \Upsilon}{\partial \tau}-\frac{t_{m} \eta}{l_{m}^{2}}\left(\frac{\partial^{2} \Upsilon}{\partial \chi^{2}}+\frac{\partial^{2} \Upsilon}{\partial \zeta^{2}}\right)=\frac{J_{0}}{l_{m} J_{m}} \mathrm{~d} \lambda \mathrm{d} \tau \delta(\chi) \delta(\zeta) \delta(\tau)
$$

Here

$$
\Upsilon=\frac{J}{J_{m}}, \quad \chi=\frac{x}{l_{m}}, \quad \zeta=\frac{y}{l_{m}}, \quad \tau=\frac{t}{t_{m}}, \quad \mathrm{~d} \lambda=\frac{\mathrm{d} l}{l_{m}}, \quad \mathrm{~d} \tau=\frac{\mathrm{d} t}{t_{m}}
$$

and the property $\delta(k x)=\delta(x) / k$ of the $\delta$-function is used.

The values of the scales $J_{m}, l_{m}, t_{m}$ can be found from the equalities

$$
\frac{t_{m} \eta}{l_{m}^{2}}=1, \quad \frac{J_{0}}{l_{m} J_{m}}=1
$$


As there are two equalities and three scales, $l_{m}$ is an arbitrary value and then

$$
t_{m}=\frac{l_{m}^{2}}{\eta}, \quad J_{m}=\frac{J_{0}}{l_{m}}
$$

The Equation (4) is presented in the dimensionless form

$$
\frac{\partial \Upsilon}{\partial \tau}-\frac{\partial^{2} \Upsilon}{\partial \chi^{2}}-\frac{\partial^{2} \Upsilon}{\partial \zeta^{2}}=\mathrm{d} \lambda \mathrm{d} \tau \delta(\chi) \delta(\zeta) \delta(\tau)
$$

Its solution at $\tau>0$ is [6] [7]:

$$
\Upsilon(\chi, \zeta, \tau)=\frac{\mathrm{d} \lambda \mathrm{d} \tau}{4 \tau} \exp \left[-\frac{\chi^{2}+\zeta^{2}}{4 \tau}\right]
$$

For $\tau<0$, that is before the momentum force operates, $\Upsilon(\chi, \zeta, \tau) \equiv 0$.

Let us introduce the dimensionless velocity

$$
v=v_{e} \frac{l_{m}}{\eta}
$$

Since $l_{m}$ is an arbitrary value, it is possible to assume $l_{m}=\eta / v_{e}$, then $v=1$.

Let at the moment of time $\tau=-N \mathrm{~d} \tau$ the elementary plate be on the abscissa at the point $-N \mathrm{~d} \lambda, N$ being an arbitrary natural number. Over the time $\mathrm{d} \tau$ it will move along the horizontal coordinate at a distance of $\mathrm{d} \lambda=\mathrm{d} \tau$, since the velocity $v=1$ and reach the point $(-N+1) \mathrm{d} \lambda$ at the moment $(-N+1) \mathrm{d} \tau$.

The process is repeated and the plate moves to the point $(-N+2) \mathrm{d} \lambda$ at the moment $(-N+2) \mathrm{d} \tau$. After $i$-jumps it gets to the point $\chi_{i}=(-N+i) \mathrm{d} \lambda$ at the moment $\tau_{i}=(-N+i) \mathrm{d} \tau$, here $i=1,2, \cdots, N$. After $N$ jumps the plate will arrive at the origin of coordinates $\chi=0$ at time $\tau=0$.

The equation of the momentum diffusion (9) for the moment $\tau_{i}$ is

$$
\frac{\partial \Upsilon}{\partial \tau}-\frac{\partial^{2} \Upsilon}{\partial \chi^{2}}-\frac{\partial^{2} \Upsilon}{\partial \zeta^{2}}=\mathrm{d} \lambda \mathrm{d} \tau \delta\left(\chi-\chi_{i}\right) \delta(\zeta) \delta\left(\tau-\tau_{i}\right)
$$

Its solution is

$$
\Upsilon\left(\chi-\chi_{i}, \zeta, \tau-\tau_{i}\right)=\frac{\mathrm{d} \lambda \mathrm{d} \tau}{4 \pi\left(\tau-\tau_{i}\right)} \exp \left[-\frac{\left(\chi-\chi_{i}\right)^{2}+\zeta^{2}}{4\left(\tau-\tau_{i}\right)}\right]
$$

Equation (13) is the diffusion momentum $\Upsilon$ produced by the elementary plate at the $i$ th moment of time.

Summarizing Equation (13) one obtains

$$
\Upsilon_{1}=\frac{\mathrm{d} \lambda}{4 \pi} \sum_{i=-N+1}^{0} \frac{1}{(\tau-i \mathrm{~d} \tau)} \exp \left[-\frac{(\chi-i \mathrm{~d} \tau)^{2}+\zeta^{2}}{4(\tau-i \mathrm{~d} \tau)}\right] \mathrm{d} \tau
$$

Equation (14) is the diffusion momentum produced by the moving elementary plate from the beginning of the moment at $\tau=-N \mathrm{~d} \tau$ up to $\tau=0$. Let us turn in (14) to a new coordinate system by the substitution

$$
\chi=\chi^{\prime}-\tau
$$

in which the plate $\mathrm{d} \lambda$ is immobile whereas the fluid is moving at a velocity of $v=-1$. 


$$
\Upsilon_{1}=\frac{\mathrm{d} \lambda}{4 \pi} \sum_{i=-N+1}^{0} \frac{1}{(\tau-i \mathrm{~d} \tau)} \exp \left[-\frac{\left(\chi^{\prime}+(\tau-i \mathrm{~d} \tau)\right)^{2}+\zeta^{2}}{4(\tau-i \mathrm{~d} \tau)}\right] \mathrm{d} \tau
$$

Further the prime is dropped. At $N \rightarrow \infty$ in (16) changing from summarizing to integrating one obtains

$$
\begin{aligned}
\Upsilon_{1}(\chi, \zeta) & =\frac{\mathrm{d} \lambda}{4 \pi} \int_{-\infty}^{\tau} \frac{1}{\tau-\tau_{1}} \exp \left[-\frac{\left(\chi+\tau-\tau_{1}\right)^{2}+\zeta^{2}}{4\left(\tau-\tau_{1}\right)}\right] \mathrm{d} \tau_{1} \\
& =\frac{\mathrm{d} \lambda}{4 \pi} \int_{0}^{+\infty} \frac{1}{s} \exp \left[-\frac{(\chi+s)^{2}+\zeta^{2}}{4 s}\right] \mathrm{d} s=\tilde{\Upsilon}(\chi, \zeta)
\end{aligned}
$$

$\tau_{1}$ changes from $-\infty$ to $\tau, s=\tau-\tau_{1}$.

The right-hand side of (17) does not contain time; it is the solutions of the stationary equation of the momentum diffusion produced by the immobile elementary plate in the moving fluid

$$
\frac{\partial \tilde{\Upsilon}}{\partial \chi}-\frac{\partial^{2} \tilde{\Upsilon}}{\partial \chi^{2}}-\frac{\partial^{2} \tilde{\Upsilon}}{\partial \zeta^{2}}=\mathrm{d} \lambda \delta(\chi) \delta(\zeta)
$$

Thus, the solution of the stationary Equation (18) is derived from the solution of the nonstationary Equation (9).

\subsection{Interaction of the Flow with the Plate}

Until now, the review did not specify the liquid or gas, since this was not necessary. Below we will consider only the flow of gas. This is due to the fact that in a gas, the molecules between collisions move independently of each other. In a liquid, there is no such independence, the molecules are constantly interacting with each other and move in clusters. These differences affect their interaction with the surface of the plate. If gas need to consider the interaction of individual molecules with a surface for fluid interaction of the cluster with it.

When a gas molecule of the mass $\mu$ runs into the plate moving at the velocity $v_{e}$, it acquires a momentum which, on the average, is

$$
\alpha \mu v_{e}
$$

and directed along the abscissa. The value of $\alpha$ specifies an average fraction of the tangential momentum imparted when the gas molecule collides with the plate surface

$$
0 \leq \alpha \leq 1
$$

From the Clapeyron-Mendeleev law it follows that the number of the gas molecules per unit of volume is

$$
n=\frac{p}{k T}
$$

Hence, the average distance between the molecules is as follows

$$
r_{0}=\sqrt[3]{\frac{1}{n}}=\left(\frac{k T}{p}\right)^{1 / 3}
$$


Here $k$ is the Boltzmann constant, $T$ is the absolute temperature, $p$ is the gas pressure. For the air $r_{0}$ is by an order of magnitude larger than the interatomic distance in condensed substances.

The molecule free path is [8]

$$
\Lambda=\frac{1}{\sqrt{2} \sigma n}=\frac{k T}{\sqrt{2} \sigma p}
$$

Here $\sigma$ is the square of the section of the molecule scattering. For the air $\Lambda \sim 10^{-7} \mathrm{~m}$.

The root-mean square velocity of the molecule thermal movement is

$$
\bar{v}=\sqrt{\frac{8 k T}{\mu}}
$$

The average time during which a molecule covers the length $\Lambda$ will be

$$
\bar{t}=\frac{\Lambda}{\bar{v}}=\frac{\sqrt{\mu k T}}{4 \sigma p}
$$

Let us consider a parallelepiped adjacent to the plate with the sides $\mathrm{d} l$ along the abscissa, $\Lambda$ along the ordinate and 1 along the $z$-axis (Figure 2 ) and call it an elementary volume. Its lower side, the plate $\mathrm{d} l$, has the square $\mathrm{d} l \times 1$. It contains the molecule number

$$
n_{\Lambda}=n \Lambda \mathrm{d} l=\frac{\mathrm{d} l}{\sqrt{2} \sigma}
$$

The sixth part of these molecules $n_{\Lambda} / 6$ move to the plate and over the time $\bar{t}$ (25) they will reach its surface. As a result of their interaction with the surface atoms of the plate, each gas molecule will get the momentum of the value (19). The total value of the momentum imparted to the molecules during the time $\bar{t}$ will be written as

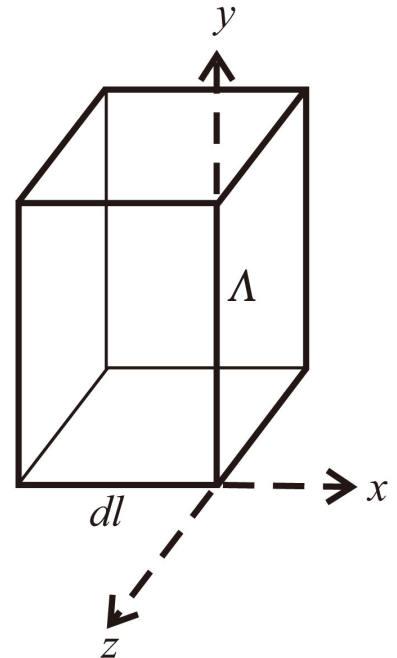

Figure 2. Shown is the elementary volume, a parallelepiped with the sides $\mathrm{d} l$ along the abscissa $x$ and $\Lambda$ along the ordinate $y$ adjacent to the plate. The length of the side along the $z$-axis is 1 . 


$$
\frac{\alpha \mu v_{e} n_{\Lambda}}{6}=\frac{\alpha \mu v_{e} n \Lambda \mathrm{d} l}{6}
$$

Equation (27) is the momentum imparted from the plate $\mathrm{d} l$ to the fluid over the time $\bar{t}$. Further that momentum will be redistributed among all the molecules $n_{\Lambda}$ of the volume $\Lambda \mathrm{d} l$ whose quantity is six times larger than that of the molecules that have interacted with the plate surface. The time of the redistribution is of the order of $\bar{t}$. As a result, each molecule, from the volume $\Lambda \mathrm{d} l$ gets, on the average, an additional diffusion momentum of the value

$$
\frac{\alpha}{36} \mu v_{e}
$$

Multipling (28) by $n_{\Lambda}$ one obtains the momentum transferred to the elementary volume from the plate over the time $\bar{t}$

$$
\frac{\alpha}{36} \mu v_{e} n_{\Lambda}
$$

Dividing (29) by $\bar{t}$ one derives the force acting on the gas volume $\Lambda \mathrm{d} l$ due to the interaction with the plate

$$
\frac{\alpha}{36} \frac{\mu v_{e} n_{\Lambda}}{\bar{t}}
$$

Let us call (30) a diffusion force, which is a volume force. Dividing (30) by the volume $\Lambda \mathrm{d} l$ one obtains the diffusion force density

$$
\frac{\alpha}{9} \frac{\sigma p^{2} v_{e}}{(k T)^{3 / 2}} \sqrt{\frac{\mu}{\pi}}
$$

Then dividing (27) by $\bar{t}$ one derives the tangential force applied to the surface of the plate $\mathrm{d} l$

$$
\frac{\alpha \mu v_{e} n \Lambda}{6 \bar{t}} \mathrm{~d} l
$$

Dividing (32) by $\mathrm{d} l$ one gets the force acting on the unit of the surface square of the plate, i.e. the tangential stress on the surface of the plate $g$ produced by the momentum exchange between the plate and the gas

$$
g=\frac{\alpha p v_{e}}{3} \sqrt{\frac{2 \mu}{\pi k T}}
$$

The force acting on the plate of the length $l$, is written as

$$
F=2 g L
$$

Since the plate has two surfaces, then the coefficient 2 is added to (34). For the formula (34) to be used correctly, it is necessary that the stress $g$ should be the same across the whole surface of the plate, i.e. the plate length $L$ should be sufficiently small. The value $F$ can be measured experimentally, which makes it possible to estimate the value $g$, and using the latter, according to (33) one can estimate the friction coefficient $\alpha$ 


$$
\alpha=\frac{3 g}{p v_{e}} \sqrt{\frac{k T}{2 \mu}}
$$

The method of calculating $g$ presented here is not suitable for a fluid, since the molecules in a fluid interact with each other directly and the notion the free path disappears. The molecules move in clusters, which represents the Brownian movement. The momentum from the plate is transferred to the fluid by the molecule clusters located near the contact of the fluid with the plate surface rather than by individual molecules as it happens in a gas. Consideration of the mechanism of the viscous interaction of a fluid with a solid is beyond the scope of this article.

In metal fluids heat conduction is caused by free electrons moving at a high velocity, whereas the momentum diffusion is produced by the molecules moving slowly. As a result, the Prandtl number for metal fluids is much less than unity.

\subsection{Plate of a Finite Size}

Let a plate be located on a segment of the axis of abscissa $(-L, 0)$. A flow is running against the plate and it is directed against the axis of abscissa with the momentum density (3) (see Figure 1), where $\mathrm{d} l$ should be replaced by $L$.

Let us consider a stationary problem. The plate $L$ is divided into the sectors $\mathrm{d} l$ numbered from the origin of the coordinates from 0 to $N$, here $N=L / \mathrm{d} l$. The number of a sector is also the number of the elementary volume related to it. A flow with the momentum density $w$ is running against the sector numbered 0 . The time during which the gas will pass the elementary volume is

$$
\frac{\mathrm{d} l}{v_{e}}
$$

and as a result, the diffusion momentum will be produced. Its value is found by multiplying the force acting on the elementary volume (30) by (36)

$$
\frac{\alpha}{36} \frac{\mu v_{e} n_{\Lambda}}{\bar{t}} \frac{\mathrm{d} l}{v_{e}}=\alpha \frac{\mu n_{\Lambda}}{36 \bar{t}} \mathrm{~d} l
$$

The momentum of the zero elementary volume of the inflowing stream $w \Lambda \mathrm{d} l$ decreases by (37) and will become

$$
w \Lambda \mathrm{d} l-\alpha \frac{\mu n_{\Lambda}}{36 \bar{t}} \mathrm{~d} l=w(1-\gamma \mathrm{d} \lambda) \Lambda \mathrm{d} l
$$

Here

$$
\gamma=\alpha \beta, \quad \beta=\frac{\eta}{36 v_{e}^{2 \bar{t}}}, \quad \mathrm{~d} \lambda=\frac{\mathrm{d} l}{l_{m}}
$$

Dividing (38) by the elementary volume $\Lambda \mathrm{d} l$ one obtains the momentum density of the flow running against the elementary volume numbered 1.

$$
w(1-\gamma \mathrm{d} \lambda)=w \mathrm{e}^{-\gamma \mathrm{d} \lambda}
$$

Here (3), (24) and $\mu n=\rho$ are used. The length $\mathrm{d} l$ can always be chosen 
small so as to satisfy $\gamma \mathrm{d} \lambda \ll 1$ and use the relation $\mathrm{e}^{-\gamma \mathrm{d} \lambda} \approx 1-\gamma \mathrm{d} \lambda$.

For the air $\eta=0.026 \mathrm{~W} / \mathrm{m} \cdot \mathrm{K}, \Lambda=10^{-7} \mathrm{~m}, \bar{v}=400 \mathrm{~m} / \mathrm{s}$ at $T=300 \mathrm{~K}$, $v_{e}=10 \mathrm{~m} / \mathrm{s}$ one obtains.

$$
\beta=29000
$$

The value $\beta \gg 1$, the value $\alpha \ll 1$ and their product (39) $\gamma \ll 1$. From mechanics, it is known that when two bodies with a different mass collide, the transferred kinetic energy is in inverse proportion to the relation of their masses [9]. When a gas molecule collides with a solid, the above effect takes place. However, since the solid mass is by orders of magnitude larger than the mass of an air molecule, probably, one should take only the mass of the volume with a diameter of the order of the phonon length rather than the whole mass of the solid. Besides, the part of the kinetic energy of the translation transferred by viscosity is small compared to the kinetic energy of the thermal movement. For instance, for the above values of the parameters the relation of the kinetic energies is $v_{e}^{2} / \bar{v}^{2}=0.0006$. The collision occurs tangentially to the solid surface, which further decreases the part of the transferred translation. As a result, when a gas molecule collides with the plate surface, the latter is given a small part of the momentum. Therefore, the value $\beta$ is so small that

$$
\gamma=\alpha \beta \ll 1
$$

The diffusion profile of the zero sector is described by the Equation (18), its solution is (17).

The flow momentum of sector 1 is given by the relation (40), the diffusion equation for it is written as

$$
\frac{\partial \Upsilon}{\partial \chi}-\left(\frac{\partial^{2} \Upsilon}{\partial \chi^{2}}+\frac{\partial^{2} \Upsilon}{\partial \zeta^{2}}\right)=\mathrm{e}^{-\gamma \mathrm{d} \lambda} \mathrm{d} \lambda \delta(\chi+\mathrm{d} \lambda) \delta(\zeta)
$$

Let us consider that $\mathrm{d} \lambda>0$, the plate is located on the abscissa $\chi$ at the point $-\mathrm{d} \lambda$. The right-hand side (43) describes the source of the diffusion momentum located at that point. The solution of (43) is

$$
\Upsilon_{1}=-\frac{\mathrm{e}^{-\gamma \mathrm{d} \lambda} \mathrm{d} \lambda}{4 \pi} \int_{0}^{\infty} \frac{1}{s} \exp \left[-\frac{(\chi+\mathrm{d} \lambda+s)^{2}+\zeta^{2}}{4 s}\right] \mathrm{d} s
$$

Adding (17) and (44) one obtains the solution (43) for a plate of the length $2 \mathrm{~d} \lambda$.

The right-hand side (43) for the $n$ sector is

$$
\mathrm{e}^{-n \gamma \mathrm{d} \lambda} \mathrm{d} \lambda(\chi+n \mathrm{~d} \lambda)(\zeta)
$$

Its solutions is

$$
\Upsilon_{n}=\frac{\mathrm{e}^{-n \gamma \mathrm{d} \lambda} \mathrm{d} \lambda}{4 \pi} \int_{0}^{\infty} \frac{1}{s} \exp \left[-\frac{(\chi+n \mathrm{~d} \lambda+s)^{2}+\zeta^{2}}{4 s}\right] \mathrm{d} s
$$

Summing up (46) with respect to $n$ and replacing summation by integration one obtains the diffusion momentum for a plate with a finite length 


$$
\begin{aligned}
\Upsilon(\chi, \zeta) & =-\frac{1}{4 \pi} \int_{-\lambda}^{0} \mathrm{e}^{-\gamma\left|\chi_{1}\right|} \int_{0}^{\infty} \frac{1}{s} \exp \left[-\frac{\left(\chi-\chi_{1}+s\right)^{2}+\zeta^{2}}{4 s}\right] \mathrm{d} s \mathrm{~d} \chi_{1} \\
& =\frac{\mathrm{e}^{-\gamma|\chi|}}{4 \sqrt{\pi}} \int_{0}^{\infty} \Psi\left[\operatorname{erf}\left(\frac{(1+2 \gamma) s+\chi+\lambda}{2 \sqrt{s}}\right)-\operatorname{erf}\left(\frac{(1+2 \gamma) s+\chi}{2 \sqrt{s}}\right)\right] \mathrm{d} s \\
& \approx \frac{\mathrm{e}^{-\gamma|\chi|}}{4 \sqrt{\pi}} \int_{0}^{\infty} \Psi\left[\operatorname{erf}\left(\frac{s+\chi+\lambda}{2 \sqrt{s}}\right)-\operatorname{erf}\left(\frac{s+\chi}{2 \sqrt{s}}\right)\right] \mathrm{d} s
\end{aligned}
$$

Here $-\left|\chi_{1}\right|=-n \mathrm{~d} \lambda$, the sign of minus is taken because the plate is located on the negative section of abscissa on the interval $(-\lambda, 0)$,

$$
\Psi=\frac{1}{\sqrt{s}} \exp \left[-\frac{\zeta^{2}}{4 s}-\gamma(1-\gamma) s\right] \approx \frac{1}{\sqrt{s}} \exp \left[-\frac{\zeta^{2}}{4 s}-\gamma s\right]
$$

Since $\gamma \ll 1$, then in (47) and (48) (47) and (48) can be neglected in comparison with unity.

Let us introduce the following symbols. If differentiation is performed with respect to the dimensional variables $x$ and $y$, then grad and div are written with small letters. If differentiation is performed with respect to the dimensionless $\chi$ and $\zeta$, then the capital letters are written - Grad, Div.

The diffusion momentum is the vector $\Upsilon=\{\Upsilon, 0\}$ with the abscissa $\Upsilon_{x}$ equal (47) and ordinate $\Upsilon_{y}=0$, its divergence is

$$
\Gamma(\chi, \zeta)=\operatorname{Div}\{\Upsilon, 0\}=\frac{\partial \Upsilon}{\partial \chi}
$$

Note the following feature of the diffusion momentum. With potential flows a change in the velocity results in a change of the pressure, and vice versa. This is not true in the case of the diffusion momentum: it is transferred by diffusion without a change of pressure or density.

\subsection{Approximation of the Quadrature Formula}

Let us consider a case when

$$
\begin{gathered}
\left|\frac{s+\chi+\lambda}{2 \sqrt{s}}\right|>c \\
\left|\frac{s+\chi}{2 \sqrt{s}}\right|>c
\end{gathered}
$$

Since $\sqrt{s}>0$, then for $c>1.7$ one can approximately assume [10]

$$
\begin{aligned}
\operatorname{erf}\left(\frac{s+\chi+\lambda}{2 \sqrt{s}}\right) & \approx \operatorname{sign}(s+\chi+\lambda) \\
\operatorname{erf}\left(\frac{s+\chi}{2 \sqrt{s}}\right) & \approx \operatorname{sign}(s+\chi)
\end{aligned}
$$

The larger $c$ the higher the accuracy of approximation (52) and (53), for instance, for $\operatorname{erf}(1.7)=0.984, \operatorname{erf}(3)=0.999978$.

To find the domains where (50) and (51) are satisfied, it is necessary to solve 
quadratic equations with respect to $\sqrt{s}$

$$
\begin{gathered}
s+2 c \sqrt{s}+\chi+\lambda=0, \quad s-2 c \sqrt{s}+\chi+\lambda=0 \\
s+2 c \sqrt{s}+\chi=0, \quad s-2 c \sqrt{s}+\chi=0
\end{gathered}
$$

The Equation (54) and Equation (55) have two similar roots

$$
\begin{gathered}
\sqrt{s}_{1,2}=-c \pm \sqrt{c^{2}-(\chi+\lambda)} \\
\sqrt{s}_{3,4}=-c \pm \sqrt{c^{2}-\chi}
\end{gathered}
$$

The sign of minus is taken from square root for indices 1 and 3 and that of plus for 2 and 4 , then with the real roots $s_{1}<s_{2}, s_{3}<s_{4}$.

At

$$
\chi>c^{2}-\lambda
$$

the Equation (54) has no real roots and (50) is satisfied for all values $0 \leq s<\infty$. In this case,

$$
s+\chi+\lambda>0
$$

Therefore,

$$
\operatorname{sign}(s+\chi+\lambda) \approx+1
$$

In (47) and (67) the substitution is used

$$
\operatorname{erf}\left(\frac{s+\chi+\lambda}{2 \sqrt{s}}\right) \approx 1
$$

Similarly, for (55) at

$$
\chi>c^{2}
$$

There are no real roots

$$
\operatorname{sign}(s+\chi) \approx+1
$$

In (47) and (67) the substitution is used

$$
\operatorname{erf}\left(\frac{s+\chi}{2 \sqrt{s}}\right) \approx 1
$$

If (62) is satisfied, then the same holds true for (58), which means that $\Upsilon=0$. Therefore, at a distance of $c^{2}$ in front of the plate the diffusion has no effect on the flow.

Squaring (57) one obtains the interval $(q, Q)$, on which approximation (53) is inadmissible

$$
\begin{gathered}
q=\left(\sqrt{s_{3}}\right)^{2}=\frac{c^{2}}{2}-\chi-\frac{c}{2} \sqrt{c^{2}-\chi} \\
Q=\left(\sqrt{s_{4}}\right)^{2}=\frac{c^{2}}{2}-\chi+\frac{c}{2} \sqrt{c^{2}-\chi}
\end{gathered}
$$

The interval for (52), where one cannot change erf for sign, is found in the same way. Under the conditions of (50), (51) it is possible to use approximation (61), (64) and (47) is written as 


$$
\Upsilon=\frac{\mathrm{e}^{-\gamma|\chi|}}{2 \sqrt{\pi}} \int_{0}^{|\chi|} \Psi \mathrm{d} s=\frac{\mathrm{e}^{-\sqrt{\gamma} \zeta-\gamma|\chi|}}{4 \sqrt{\gamma}}\left[\operatorname{erfc}\left(\frac{\zeta-2 \sqrt{\gamma}|\chi|}{2 \sqrt{|\chi|}}\right)-\mathrm{e}^{2 \sqrt{\gamma \zeta}} \operatorname{erfc}\left(\frac{\zeta+2 \sqrt{\gamma}|\chi|}{2 \sqrt{|\chi|}}\right)\right]
$$

Equation (67) does not include the plate length $\lambda$. Equation (67) gives the correct values of $\Upsilon$ for the interval $\chi \in\left(-\lambda+c^{2},-c^{2}\right)$. Outside the above interval, one should use (47) where the plate length $\lambda$ comes into play. This is illustrated in Figure 3, which shows the plots of the diffusion momentum $\Upsilon$ for $\gamma=0.0001$ of a plate with the length $\lambda=1 / \gamma=10^{4}$ calculated according to (47) - (a) and (67) - (b). They coincide. For $\chi \in\left(-\lambda+c^{2},-c^{2}\right)$ the calculation is possible according to (67).

Maximum $\Upsilon$ at the given $\chi$ is located on the surface of the plate, where $\Upsilon$ increases from 0.3 for $\chi=0$ up to 21 at $\chi \sim-5000$, with $\Upsilon$ further decreasing at a distance from the plate. When a distance of $\zeta \sim 100 \Upsilon$ is reached, $\Upsilon$ is close to zero. The diffusion momentum $\Upsilon$ is the deceleration momentum. The larger $\Upsilon$ the slower the flow moves and the weaker its effect on the plate, which, in turn, produces less diffusion momentum. This is clearly seen in Figure 3. At first the deceleration value on the plate surface increases quickly (the curves $1,2,3$ for the interval $(-2500,0))$. Then $\Upsilon$ remains approximately constant on the interval $(-5000,-2500)$. After that the slope of the $\Upsilon$ curve becomes more flattened, which suggests that the diffusion rate decreases, as it is proportional to the slope. It is the decrease in the slope that indicates stabilization in the $\Upsilon$ value on the plate surface, since the less diffusion momentum is

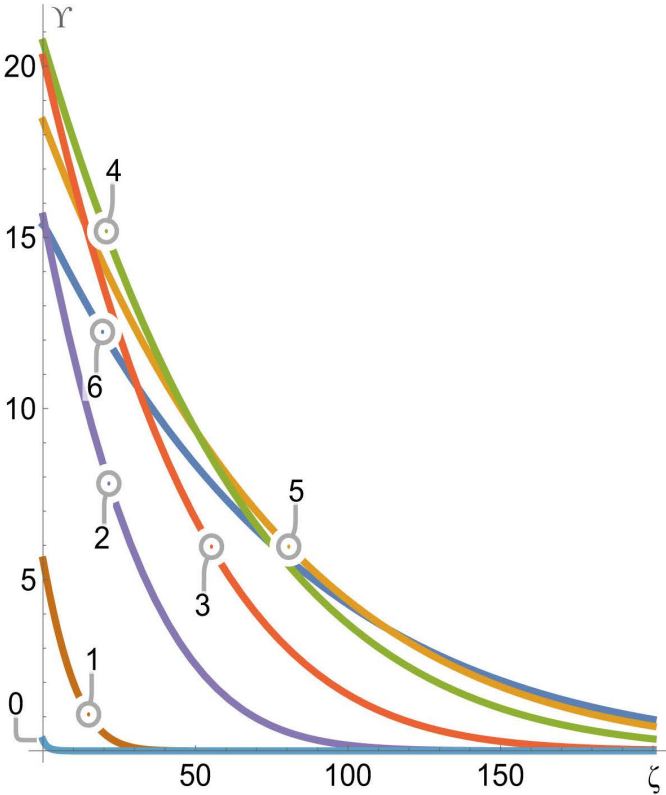

(a)

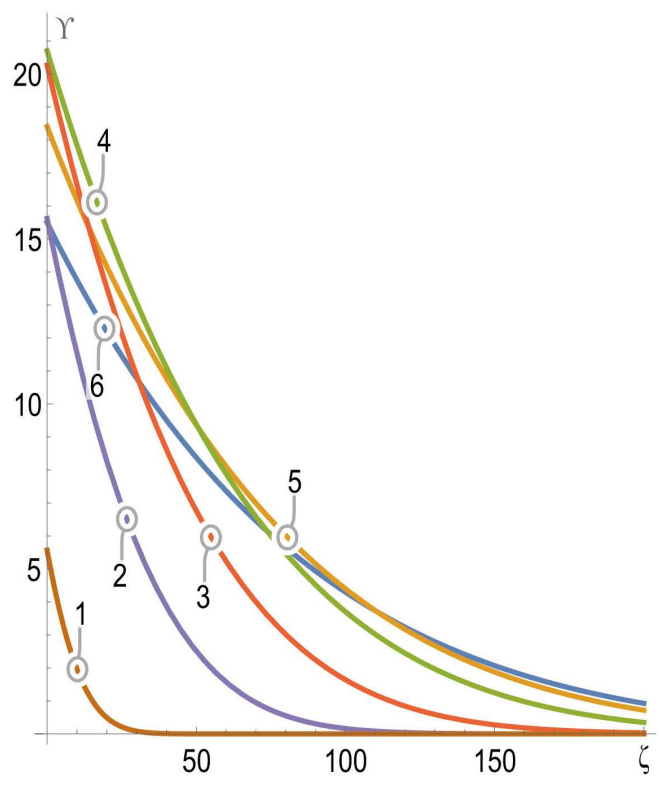

(b)

Figure 3. The dependence of $\Upsilon(\chi, \zeta)$ for $\gamma=0.0001$ on $\zeta$ for abscissa values: 0$) \quad \chi=0,1)$ $\chi=-100,2) \quad \chi=-1000,3) \quad \chi=-2500,4) \quad \chi=-5000,5) \quad \chi=-7500,6) \quad \chi=-10000$, calculated according to the formulas (47) for a) and (67) for (b). The formula (67) is not suitable for calculation at $\chi=0$. Therefore, in the figure (b) there is no profile for $\chi=0$. 
produced the less of it is further transmitted. After the point $\chi=-5000$ is reached, the momentum value becomes so low that $\Upsilon$ on the plate surface decreases.

$1 / \gamma$ is the characteristic length of the plate, with the plate length $\lambda \sim 1 / \gamma$ the profiles of $\Upsilon$ being similar. Figure 4 shows the $\Upsilon$ plots at $\gamma=0.001$ for $\lambda=1000$. These plots are similar to those in Figure 3.

Figure 5 demonstrates the diffusion profile of $\Upsilon$ behind the plate (the wake) for $\lambda \sim \chi=-\lambda,-1.1 \lambda,-1.2 \lambda,-1.5 \lambda$ for $\lambda=1000$ calculated according to (47).

The interval of change of $\zeta$ in Figure 5 includes the negative values of $\zeta$. In the plot for $\chi=-\lambda$ one can see a bend caused by the presence of the momentum source on the plate surface. Behind the plate, it disappears smoothed by the diffusion. Here we have considered the case of a plate flown around by a stream without the formation of eddies.

\subsection{Induced Field}

The momentum diffusion field (47) is the first approximation of the solution of the plate flown around problem. It creates volume sources of momentum, which is equivalent to the appearance of a volume force in the flow. The second approximation is the calculation of the velocity field created by these volume sources, which we will call the induced field. The vectors $\boldsymbol{T}, \boldsymbol{U}, \boldsymbol{V}$ are the dimensionless induced momenta.

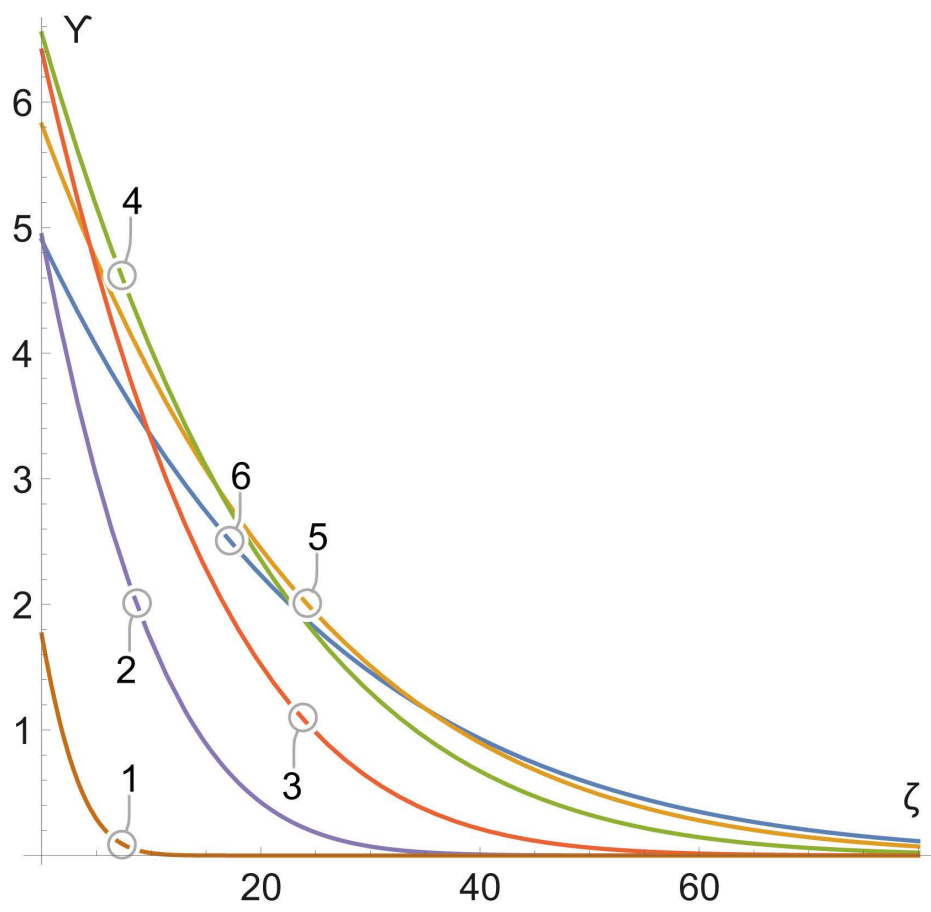

Figure 4. Shown are the diffusion profiles $\Upsilon(\chi, \zeta)$ for $\lambda=1000$ for the following abscissa values: 1) $\chi=-10,2) \quad \chi=-100,3) \quad \chi=-250,4) \quad \chi=-500,5) \quad \chi=-750,6)$ $\chi=-1000$. 


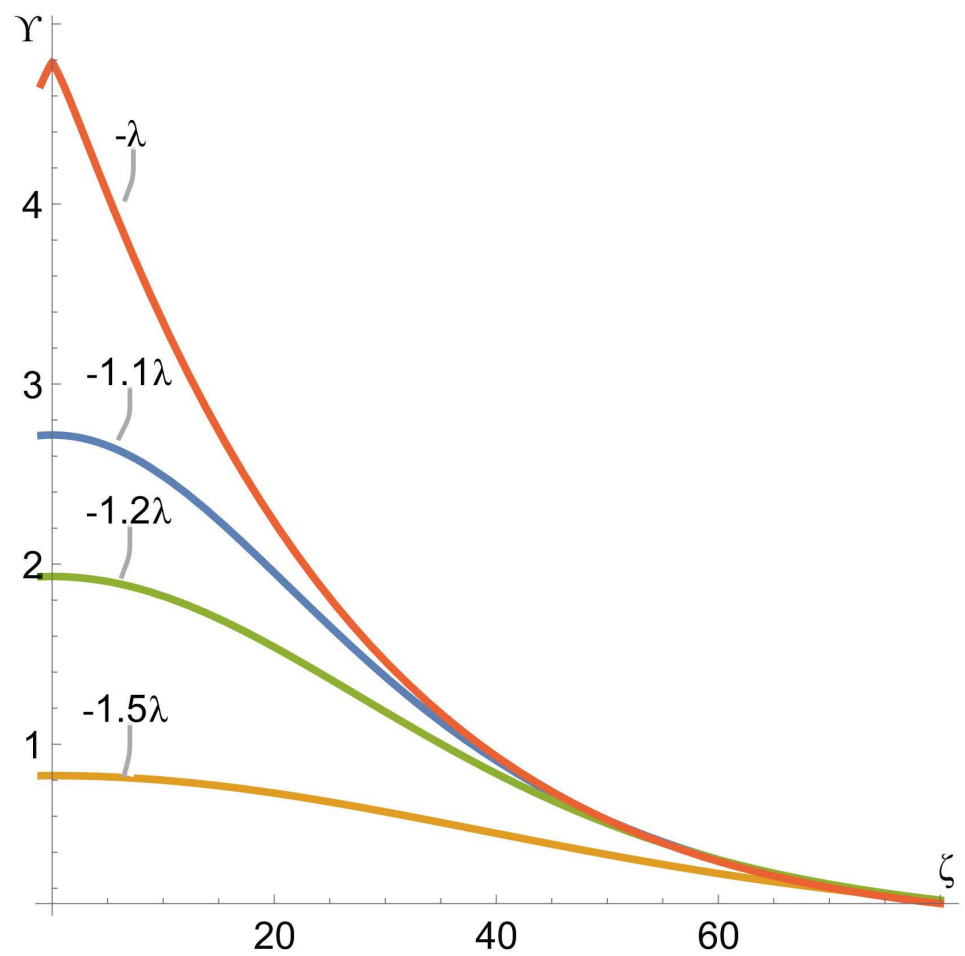

Figure 5. Shown are the profiles of the diffusion momentum in the wake behind the plate with the dimensionless length $\lambda=1000$ for the points $\chi=-\lambda,-1.1 \lambda,-1.2 \lambda,-1.5 \lambda$. There is a bend in the plot at the point $\zeta=0$ passing through the point $\chi=-\lambda$ of the plate. In the other plots, there is no bend.

Substitution of (67) into (49) shows that $\Gamma \neq 0$, which means that the momentum diffusion produces volume momentum sources without a mass influx.

The total momentum density in dimensionless variables is

$$
\boldsymbol{w}+\boldsymbol{J}+\boldsymbol{T}
$$

The continuity equation for $\boldsymbol{T}$ is

$$
\operatorname{Div} \boldsymbol{T}=\Gamma
$$

The potential solution for (69) is

$$
\boldsymbol{U}(\chi, \zeta)=\operatorname{Grad} \mathbb{P}
$$

The potential $\mathbb{P}$ is

$$
\mathbb{P}=\frac{1}{2 \pi} \Gamma * \ln \sqrt{\chi^{2}+\zeta^{2}}
$$

The symbol $*$ denotes convolution. The potential velocity field is

$$
\boldsymbol{U}=\operatorname{Grad} \mathbb{P}=\frac{1}{2 \pi} \Gamma(\chi, \zeta) * \frac{1}{\left(\chi^{2}+\zeta^{2}\right)}\{\chi, \zeta\}
$$

Figure 6 shows the plots of the potential abscissa and ordinate components $\boldsymbol{U}$ for $\chi=50, \chi=100, \chi=2500, \chi=5000$. Their value is by an order of magnitude less than that of $\Upsilon$ (Figure 3 ). The potential momentum has the largest value near the front edge of the plate. 


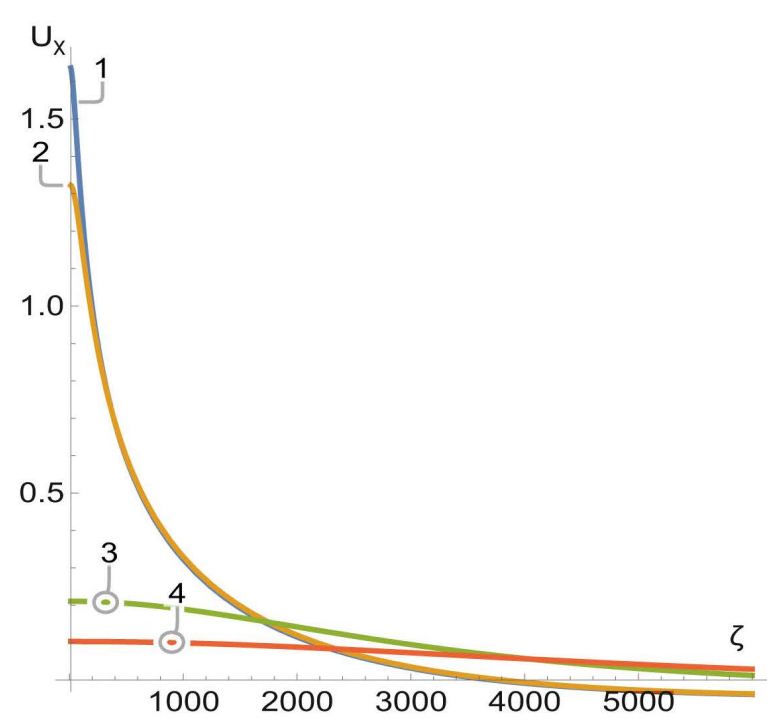

(a)

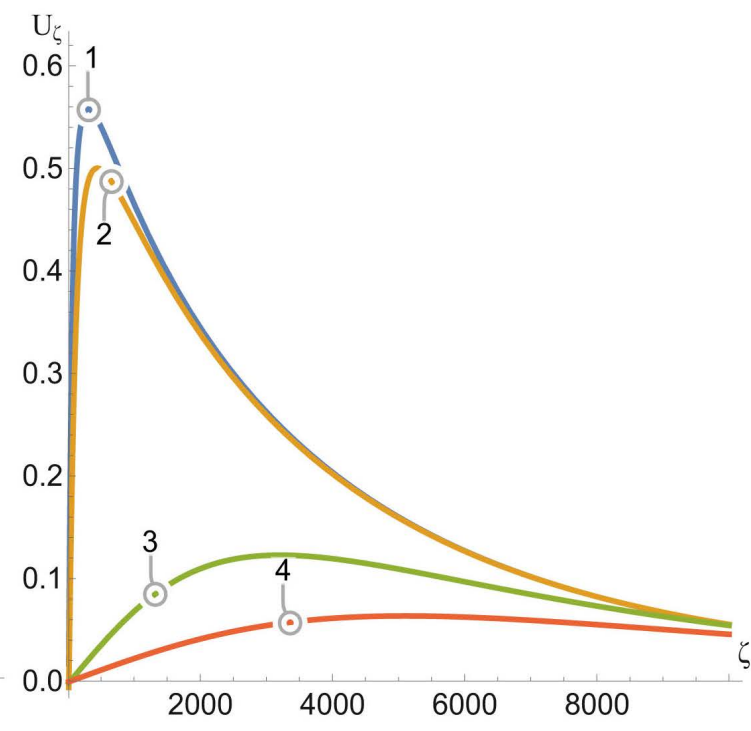

(b)

Figure 6. The potential component $\boldsymbol{U}$ versus $\zeta$ for 1) $\chi=50,2) \quad \chi=100,3) \quad \chi=2500,4) \quad \chi=2500$. (a) The potential component $U_{\chi}$. (b) The potential component $U_{\zeta}$.

The nonpotential solution $\boldsymbol{V}$ is

$$
\boldsymbol{V}=\frac{1}{4} \Gamma(\chi, \zeta) * \frac{3 \chi^{2}+\zeta^{2}}{\left(\chi^{2}+\zeta^{2}\right)^{2}}\{\chi, \zeta\}
$$

Figure 7 shows the plots of the nonpotential solution $\boldsymbol{V}$ for the points: 1) $\chi=50,2) \quad \chi=100,3) \quad \chi=1000,4) \quad \chi=2500, \quad \chi=5000$.

The general solution is written as follows

$$
\boldsymbol{T}=c_{1} \boldsymbol{U}+c_{2} \boldsymbol{V}
$$

Here $c_{1}, c_{2}$ are the arbitrary constants. Substituting $\boldsymbol{T}$ into (69) one obtains

$$
c_{1} \operatorname{Div} \boldsymbol{U}+c_{2} \operatorname{Div} \boldsymbol{V}=\Gamma
$$

The $\Upsilon$ maximum is considerably larger than the maximum of the induced components $\boldsymbol{U}, \boldsymbol{V}$ as can be seen from the plots in Figure 3, Figure 6 and Figure 7. The induced components decay slower than $\Upsilon$. For instance, $\Upsilon$ becomes small at a distance of 100 dimensionless units, whereas the induced components at a distance of 2000 and more than that. Let us call the domain of the considerable values of the diffusion momentum $\Upsilon$ the body of the boundary layer, and the domain of the considerable values of the induced components the mantle. The third region, a viscous sublayer, is described below. It is by an order of magnitude thinner than the body of the boundary layer, with the diffusion force $f$ being significant there.

Thus, in dimensional variables, the thickness of the boundary layer body is $100 l_{m}$, and the thickness of the mantle is $2000 l_{m}$.

To find $c_{1}$ and $c_{2}$, one needs two additional conditions. First, for any point $\left(\chi_{0}, \zeta_{0}\right)$ the momentum flow through the closed contour around it is equal to 


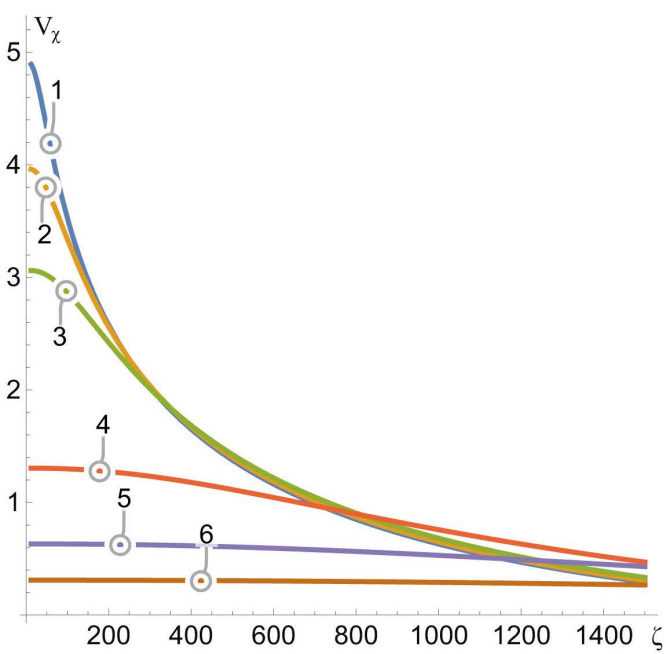

(a)

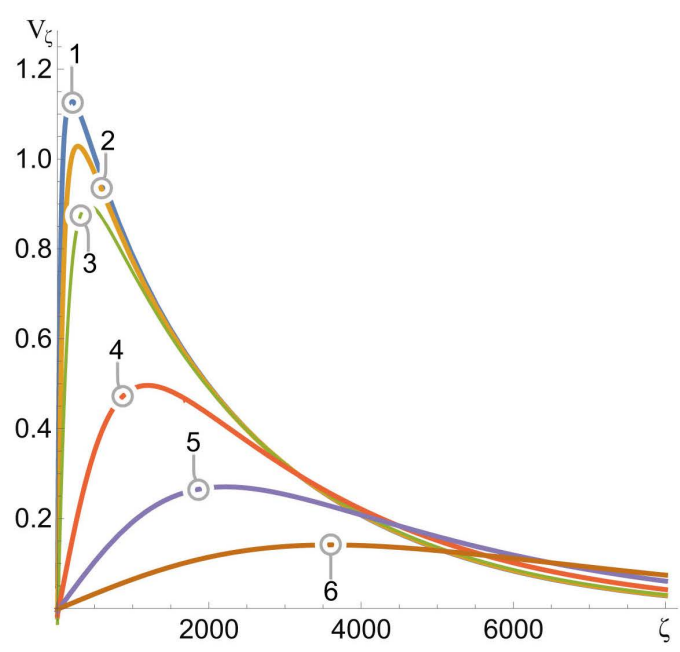

(b)

Figure 7. Plots of the dependence of the nonpotential induced component $\boldsymbol{V}$ on $\zeta$ for: 1) $\chi=50,2$ ) $\chi=100,3) \quad \chi=200,4) \quad \chi=1000,5) \quad \chi=2500,6) \quad \chi=5000$. (a) The nonpotential component $V_{\chi}$. (b) The nonpotential component $V_{\zeta}$.

the momentum produced by the sources inside the contour. The above condition excludes one arbitrary constant, e.g. $c_{2}$. To find $c_{1}$, one needs the second condition, e.g. the minimum energy of the system.

The flow of the vectors $\boldsymbol{U}, \boldsymbol{V}$ through the circle of the radius $R$ with the center at the point $\chi_{0}, \zeta_{0}$ is written as

$$
\begin{aligned}
G_{U} & =\int_{0}^{2 \pi} U_{R} R \mathrm{~d} \varphi \\
G_{V} & =\int_{0}^{2 \pi} V_{R} R \mathrm{~d} \varphi
\end{aligned}
$$

Here $U_{R}, V_{R}$ are the radial components of the vectors $\boldsymbol{U}, \boldsymbol{V}$ in the polar system of the coordinates with the centre at the point $\chi_{0}, \zeta_{0}$. The transition is achieved through the change of the coordinates $\boldsymbol{U}, \boldsymbol{V}$ in the polar system of the coordinates with the centre at the point $\chi_{0}, \zeta_{0}$. The transition is achieved through the change of the coordinates

$$
\chi^{\prime}=R \cos \varphi, \quad \zeta^{\prime}=R \sin \varphi
$$

Let us expand $\Gamma(\chi, \zeta)$ in (72) and (73) into the Tailor series near the point $\chi_{0}, \zeta_{0}$ about the degrees $\chi^{\prime}, \zeta^{\prime}$ and calculate a flow produced by the zero component in the expansion $\Gamma_{0}=\Gamma\left(\chi_{0}, \zeta_{0}\right)$. The formulas (72) and (73) look like

$$
\begin{gathered}
\boldsymbol{U}=\frac{\Gamma_{0}}{2 \pi} \iint_{S_{R}} \frac{1}{\left(\chi^{\prime}-\chi_{1}\right)^{2}+\left(\zeta^{\prime}-\zeta_{1}\right)^{2}}\left\{\chi^{\prime}-\chi_{1}, \zeta^{\prime}-\zeta_{1}\right\} \mathrm{d} \chi_{1} \mathrm{~d} \zeta_{1} \\
\boldsymbol{V}=\frac{\Gamma_{0}}{4 \pi} \iint_{S_{R}} \frac{3\left(\chi^{\prime}-\chi_{1}\right)^{2}+\left(\zeta^{\prime}-\zeta_{1}\right)^{2}}{\left[\left(\chi^{\prime}-\chi_{1}\right)^{2}+\left(\zeta^{\prime}-\zeta_{1}\right)^{2}\right]^{2}}\left\{\chi^{\prime}-\chi_{1}, \zeta^{\prime}-\zeta_{1}\right\} \mathrm{d} \chi_{1} \mathrm{~d} \zeta_{1}
\end{gathered}
$$


Integration in (73) is performed in the circle $S_{R}$ of the radius $R$ with the centre at the point $\chi_{0}, \zeta_{0}$.

Let us turn to the polar coordinates

$$
\chi^{\prime}=R \cos \varphi, \quad \zeta^{\prime}=R \sin \varphi
$$

The radial components of the induced vectors on the circle are written as

$$
\begin{gathered}
U_{R}=\Gamma_{0} \iint_{S_{R}} q_{U} \mathrm{~d} \chi_{1} \mathrm{~d} \zeta_{1} \\
V_{R}=\Gamma_{0} \iint_{S_{R}} q_{V} \mathrm{~d} \chi_{1} \mathrm{~d} \zeta_{1}
\end{gathered}
$$

Here it is designated

$$
\begin{gathered}
q_{U}=\frac{R-\chi_{1} \cos \varphi-\zeta_{1} \sin \varphi}{2 \pi\left[R^{2}+\chi_{1}^{2}+\zeta_{1}^{2}-2 R\left(\chi_{1} \cos \varphi+\zeta_{1} \sin \varphi\right)\right]} \\
q_{V}=\frac{\left[3\left(R \cos \varphi-\chi_{1}\right)^{2}+\left(R \sin \varphi-\zeta_{1}\right)^{2}\right]\left(R-\chi_{1} \cos \varphi-\zeta_{1} \sin \varphi\right)}{4 \pi\left[R^{2}+\chi_{1}^{2}+\zeta_{1}^{2}-2 R\left(\chi_{1} \cos \varphi+\zeta_{1} \sin \varphi\right)\right]^{2}}
\end{gathered}
$$

Integrating (84) and (85) with respect to the contour $S_{R}$ one obtains

$$
\begin{aligned}
& \int_{0}^{2 \pi} q_{U} R \mathrm{~d} \varphi=\frac{1}{2} \\
& \int_{0}^{2 \pi} q_{V} R \mathrm{~d} \varphi=\frac{1}{2}
\end{aligned}
$$

The flow of the induced vectors through the circle $S_{R}$ according to (76) and (77) is written as

$$
\begin{aligned}
G_{U} & =\Gamma_{0} \iiint_{S_{R}}^{2 \pi} q_{0}^{2 \pi} q_{U} R \mathrm{~d} \varphi \mathrm{d} \chi_{1} \mathrm{~d} \zeta_{1}=\frac{\Gamma_{0}}{2} \iint_{S_{R}} \mathrm{~d} \chi_{1} \mathrm{~d} \zeta_{1}=\frac{\pi}{2} R^{2} \Gamma_{0} \\
G_{V} & =\Gamma_{0} \iint_{S_{R}}^{2 \pi} \int_{0}^{2 \pi} q_{V} R \mathrm{~d} \varphi \mathrm{d} \chi_{1} \mathrm{~d} \zeta_{1}=\frac{\Gamma_{0}}{2} \iint_{S_{R}} \mathrm{~d} \chi_{1} \mathrm{~d} \zeta_{1}=\frac{\pi}{2} R^{2} \Gamma_{0}
\end{aligned}
$$

Since

$$
\iint_{S_{R}} \mathrm{~d} \chi_{1} \mathrm{~d} \zeta_{1}=R^{2}
$$

dividing (88) and (89) by (90) one derives the value of the divergence produced by the induced momenta $\boldsymbol{U}$ and $\boldsymbol{V}$ at the point $\chi_{0}, \zeta_{0}$ in the form

$$
\operatorname{Div} \boldsymbol{U}=\operatorname{Div} \boldsymbol{V}=\frac{1}{2}
$$

Substituting the values of (91) into (75) and taking into account the fact that the value $\Gamma$ in the right-hand side of (75) should also be taken at the point $\chi_{0}, \zeta_{0}$ one obtains

$$
c_{2}=2-c_{1}
$$

Hence and from (74):

$$
\boldsymbol{T}=c_{1} \boldsymbol{U}+\left(2-c_{1}\right) \boldsymbol{V}
$$


The above relation contains one arbitrary constant $c_{1}$, its value can be defined from the condition of the minimum of energy flow. This problem calls for a separate consideration and it is beyond the scope of the present article.

\section{How Eddies Are Generated}

The emergence of eddies when a viscous fluid flows around a plate has not been thoroughly studied in theory. That they emerge in a fluid or a gas is explained by the action of the tangential viscous stresses which do not exist in nature. The problem is not trivial. The formation of an eddy requires the momentum of force. However, the question about how viscous stresses produce the momentum of force has not been answered yet. Besides, the simplest experiments reject this hypothesis. If one takes a glass with water and begins to rotate it, one can make sure that to make the water rotate in the glass due to viscosity, one has to rotate it for a long time. However, it should be noted that eddies form without the rotation of a plate or any external flow. In a fluid or a gas the surface forces can have only components normal to the surface, as it happens with pressure, i.e. there are no shear stresses. This is how they are different from a solid.

If the work of the force on a closed contour is not zero, then it produces the momentum of force. There can be no rotation without it. Therefore, the main problem of the formation of an eddy is the nature of the momentum of force.

\section{Diffusion Force}

The total derivative of the diffusion momentum density, i.e. the diffusion force is

$$
F=\frac{\mathrm{d} J}{\mathrm{~d} t}=\frac{\partial J}{\partial t}+\frac{J}{\rho} \frac{\partial J}{\partial x}
$$

For the stationary case (94) changes over to

$$
F=\frac{J}{\rho} \frac{\partial J}{\partial x}=\frac{J_{m}^{2}}{\rho l_{m}} \Upsilon(\chi, \zeta) \frac{\partial \Upsilon(\chi, \zeta)}{\partial \chi}=\frac{g^{2}}{\rho v_{e} \eta} f
$$

This force has only abscissa - component $F_{x} \neq 0$ directed against the flow, ordinate - component $F_{y} \equiv 0$. This is also the case for $\boldsymbol{f}=\{f, 0\}$. Here

$$
f(\chi, \zeta)=\Upsilon(\chi, \zeta) \frac{\partial \Upsilon(\chi, \zeta)}{\partial \chi}
$$

Let $f$ depend linearly on $\zeta$

$$
f=k\left(\zeta-c_{0}\right)=k \zeta_{1}
$$

Here $\zeta_{1}=\zeta-c_{0}$. The function $f\left(\chi, \zeta_{1}\right)$ can be expanded into the Tailor series near the point $\chi, c_{0}$, with the coefficient at power one $\zeta_{1}$ being

$$
k=\frac{\partial f}{\partial \zeta}\left(\chi, c_{0}\right)
$$

or it is possible to linearize $f$ using a different approximation; its specific form is not significant.

In the polar coordinates $\varrho, \varphi$ 


$$
\chi=\varrho \cos \varphi, \quad \zeta_{1}=\varrho \sin \varphi
$$

with the centre at the point $\left(\chi, c_{0}\right)$ the azimuth component of the vector $f$ is represented in this way

$$
f_{\varphi}=-k \zeta_{1} \sin \varphi=-k \varrho \sin ^{2} \varphi
$$

The moment of force in the dimensional form $\mathrm{d} M$, with respect to the point $\left(\chi, c_{0}\right)$ produced by the force $\boldsymbol{f}$ in the radius ring $\varrho$ with the thickness $\mathrm{d} \varrho$ is the written as

$$
\mathrm{d} M=\frac{g^{2}}{\rho v_{e} \eta} l_{m}^{3} \mathrm{~d} \varrho \int_{0}^{2 \pi} f_{\varphi} \varrho^{2} \mathrm{~d} \varphi=-\pi k \frac{g^{2} \eta^{2}}{\rho v_{e}^{4}} \varrho^{3} \mathrm{~d} \varrho
$$

Figure 8 shows the dependence of the force $f(96)$ at $\gamma=0.0001$. The diffusion force is concentrated within the interval of 20 - 30 dimensionless units near the plate, which will be called a viscous sublayer. In dimensional variables, the thickness of the viscous sublayer will be $20-30 l_{m}$. It is much thinner than the boundary layer. It is there that eddies are generated. Within the interval of $-2000<\chi<0$ near the plate there exist sections with practically linear dependence $\Upsilon(\zeta)$, where the moment of force is produced. Linearisation of these plots gives the values of $k=-0.080,-0.054,-0.033,-0.017,-0.010,-0.006$. The coefficient order corresponds to the numbers of the plots in Figure 8 . The coefficients are negative, so taking into account the minus with the azimuth component, in (100), one obtains the positive azimuth component, and, hence, the moment of force produced by it is positive and directed counter clockwise. The eddy seems to roll along the surface of the plate in the direction of the flow. The above direction of rotation agrees with the observation data on the eddies produced when the plate is flown around by a stream. Besides, the above direction of the moment produces the pressing effect of the flow to the plate. Since the point of the maximum deceleration is located on the plate, the direction of the rotation along the flow as if rolls the eddy in the direction of the movement of the flow. If the rotation is in the opposite direction, it obstructs the movement of the flow along the plate so the eddy "rolls" against the flow breaking it off the surface of plate. In the curve 7 of Figure 8 there is a section near the plate with the positive value of the angle of the slope, that is the place where the negative moment of force starting to rotate the fluid clockwise. From that moment eddies break off the surface of the plate and the laminar flow turns into the turbulent one.

At $\chi=-2000$ the curve begins to bend and at $\chi=-3000$, there appears a section in the plot in which $\partial \mathrm{r} / \partial \zeta>0$, i.e. there appears a region where rotation occurs in the negative direction. It results in the eddy breaking off the plate surface and the beginning of turbulence. Thus, the dimensionless length of the plate $\lambda_{*} \sim 2000$ for $\gamma=0.0001$ is the critical length of the transition to turbulence. In the dimensional form this relation looks like

$$
\lambda_{*}=\frac{L}{l_{m}}=\frac{v_{e} L}{\eta} \sim 2000
$$




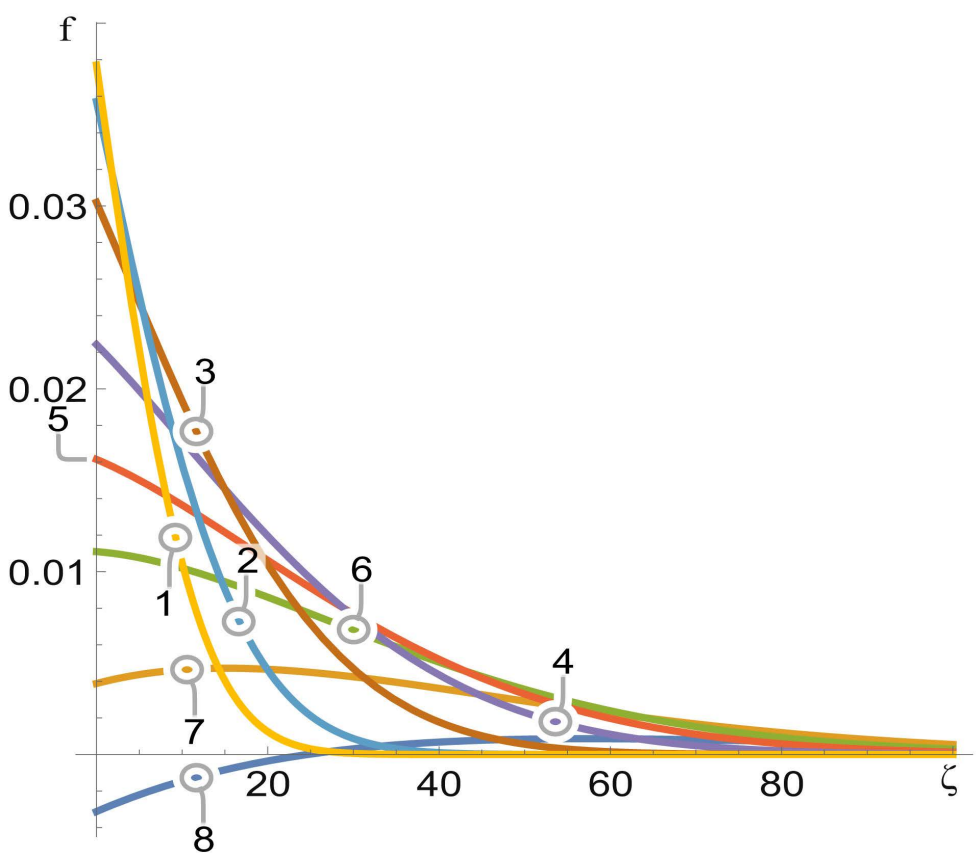

Figure 8. Plots of the dependences of the diffusion force $f$ on $\zeta$ at the points $\chi=1$ ) $-100,2)-200,3)-500,4)-1000,5)-1500,6)-2000,7)-3000,8)-5000$ at $\gamma=0.0001$.

The value

$$
\mathrm{Re}=\frac{v_{e} L}{\eta}
$$

is the Reynolds number. The consideration presented here gives the correct value of the Reynolds number for the transition of the laminar flow to the turbulent one at $\gamma=0.0001$. For small values of $\gamma$ the critical dimension of $\lambda_{*}$ is larger, at present $\gamma$ is not measured.

The inertia moment of the ring is written as

$$
\mathrm{d} Z=2 \pi \rho l_{m}^{4} \varrho^{3} \mathrm{~d} \varrho=2 \pi \rho \frac{\eta^{4}}{v_{e}^{4}} \varrho^{3} \mathrm{~d} \varrho
$$

From (101) and (104) it follows that with linear dependence of the volume force density on the coordinate it produces angular acceleration independent of the radius of the ring.

$$
\dot{\omega}=\frac{\mathrm{d} M}{\mathrm{~d} Z}=-\frac{k}{2} \frac{g^{2}}{\rho^{2} \eta^{2}}
$$

Under the action of the moment (101) the fluid volume rotates like a solid with the same angular velocity at all points.

Estimation. Let $g=1 \mathrm{~N} / \mathrm{m}^{2}, \rho=2 \mathrm{~kg} / \mathrm{m}^{3}, \eta=10^{-5} \mathrm{~m}^{2} / \mathrm{s}, k=10^{-2}$, then

$$
\dot{\omega} \sim 10^{7} \mathrm{~s}^{-2}
$$

The eddy diameter is of the order of the thickness of the viscous sublayer. The eddies really observed have a diameter that is much larger - of the order of the thickness of the body of the boundary layer and more than that. Besides, the an- 
gular velocity (106) turns out larger. If the plate length is $\sim 0.1 \mathrm{~m}$, then the time it takes the flow to pass at the velocity $v_{e} \sim 10 \mathrm{~m} / \mathrm{s}$ will be $0.01 \mathrm{~s}$. During this time the eddy in the viscous sublayer has to reach the angular velocity $\omega \sim 10^{5} \mathrm{~m} / \mathrm{s}$. Due to the momentum diffusion, this angular velocity will relax. The inertia moment of the cylinder of the radius $r$ relative to its axis per unit length along the $z$-coordinate is [9]

$$
\frac{\pi}{2} \rho r^{4}
$$

Therefore, the increase in the cylinder radius $r$ ten times with the same momentum results in the decrease of the angular velocity $10^{4}$ times, i.e. the angular velocity $\omega$ will decrease from $10^{5} \mathrm{~s}^{-1}$ down to $10 \mathrm{~s}^{-1}$. The time necessary for the distribution of the momentum moment of the cylinder with the radius $30 l_{m}$ onto that with the radius $300 l_{m}$ is

$$
\frac{\left(300 l_{m}\right)^{2}}{\eta} \sim 10^{5} \frac{\eta}{v_{e}^{2}} \sim 0.01 \mathrm{~s}
$$

This is the time of covering a distance equal to the length of the plate.

Attraction of eddies. Theoretically, the rotation of two cylinders in a viscous liquid is discussed [11] [12], but we are not interested in their interaction with a viscous liquid medium, but they are used exclusively to illustrate the joining (summation) of vortices.

In addition to diffusion, the distribution of the momentum can follow the mechanism of "summation". Let there be two rotating cylinders with the radius $R$ and the angular velocity $\omega$ (Figure 9 ).

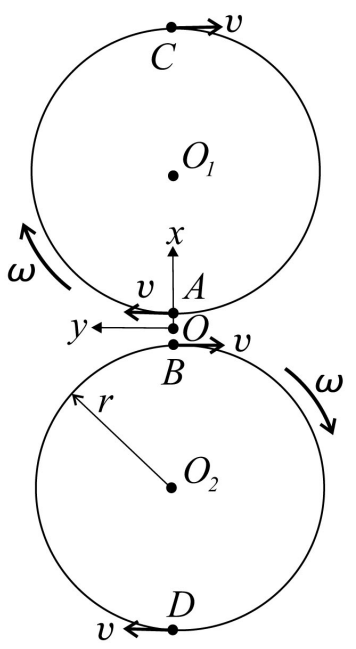

(a)

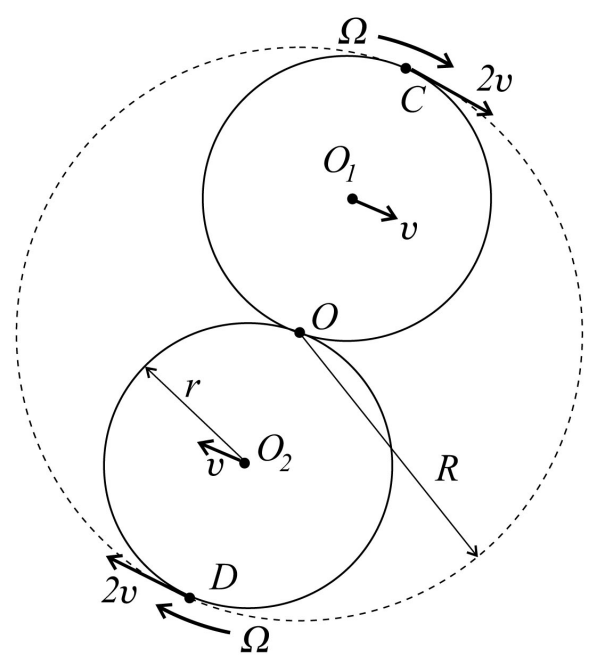

(b)

Figure 9. In the figure (a) one can see two solid-state cylinders rotating at the same velocity $\omega$ around the points $O_{1}$ and $O_{2}$. On engagement at point $O$ they begin to rotate around it as shown in the figure (b). The broken-line circle denotes the trajectory of the movement of the points $C$ and $D$ after engagement. The azimuth velocity of the points $O_{1}$ and $\mathrm{O}_{2}$ is denoted by $v$ and the angular velocity of the combined rotation of the cylinders around the axis $O$ is designated by $\Omega$. 
The moment of momentum and the kinetic energy for the rotating cylinder in Figure $9(a)$ is written as

$$
\begin{aligned}
& \frac{\pi}{2} \rho \omega r^{4} \\
& \frac{\pi}{4} \rho \omega^{2} r^{4}
\end{aligned}
$$

On the engagement of the cylinders, there happens a transition from the individual rotation (a) to the combined one (b), with the moment of momentum retained and the excess energy leaving for the environment and heating the cylinders. The moment of inertia of the rotation of the cylinder around the axis $O$ is

$$
\frac{3 \pi}{2} \rho r^{4}
$$

The moment of momentum for the rotation of the cylinder around the axis $O$ is

$$
\frac{3 \pi}{2} \Omega \rho r^{4}
$$

Equating (112) to (109) one derives $\Omega=\omega / 3$. The kinetic energy for (b) is

$$
\frac{\pi}{12} \rho \omega^{2} r^{4}
$$

Comparing (110) and (113) one obtains the kinetic energy for the variant (a) three times as much as that for the variant (b) with the similar moment of momentum. The rotation variant (b) is more preferable than (a) as regards to energy. Thus, if a fluid has several eddies, they will tend to combine with a decrease in the angular velocity.

Stability of the circular motion of a fluid. During the rotation of a circular ring the pressure inside it is the same, which holds true for its external part, so the pressure on its external boundary will be the same at all points of the external contour. The difference in the pressures on the internal and external contours gives rise to the centripetal force. The circular motion of a fluid will be stable. If a rotating fluid ring changes into an ellipse whose curvature is not stable, the centripetal force will be unstable either. Where the curvature is larger the difference in the pressure between the internal and the external contours of the ring is larger, and so is the centripetal force. As a result, the pressure inside the elliptical ring will be unstable and it will be smaller with the arcs of a larger curvature and vice versa. It will make the fluid moving inside the ring equilibrate the pressure, which finally leads to a change of the elliptical ring into the circular one. Therefore, the elliptical rotation is unstable and it changes over to the circular one. If due to the action of the volume force, there arises the motion of a fluid rotating along arbitrary trajectories, it evolves into circular eddies.

\section{Summary}

In the dynamics of viscous liquids and gases, the concept of viscous stresses is 
used. These stresses are by definition tangents. Tangential stresses can only exist in a solid. They don't exist in liquids and gases. Their use leads the theory of viscous fluid flow to a dead end. The theory is not able to give a correct quantitative description of the observed effects. To adequately describe the flow of a viscous medium, it is necessary to include the momentum diffusion equation, which is currently not included in the system of Euler equations describing hydrodynamic flow. Momentum diffusion is the main process that determines the main parameters of a viscous flow, and the diffusion equation provides a detailed description of the viscous flow process. For viscosity, this equation turns out to be the main one. Without it, it is impossible to calculate the flow parameters of both laminar and turbulent flow. It is the momentum diffusion that leads to the formation of vortices in the wake during laminar flow around bodies, to the separation of the laminar flow from the streamlined surface and the transition to a turbulent flow.

The "sticking" condition is used as a boundary condition in the equation of motion when the plate flows around, that is, the velocity on the surface of the plate is considered to be zero. This is not an experimental fact, it is a postulate accepted without serious justification. The sticking condition for certain types of flow is absurd. For example, the flow of a ceramic surface with a noble gas, argon. The surface of the argon atoms is not captured, there is no sticking. An incorrect boundary condition leads to solutions that contradict the observed effects. The analysis carried out here uses the kinetic interaction of a gas with a streamlined surface, and the dependence of the surface interaction parameters on the thermodynamic parameters of the flowing gas is found out. This is necessary for creating experimental methods for measuring the parameters of interaction between a gas and a streamlined surface.

\section{Conclusions}

Viscosity is the result of the momentum diffusion. Viscous stresses do not exist in nature. In [1], the author considers the momentum diffusion without taking into account the deceleration of the flow along the plate. The problem discussed there is related to the case of short plates, in which the slow-down effect does not work. In the present article, the author has taken into account the deceleration effect. The solution for the diffusion momentum has been found in the explicit form. It describes the effects observed under a viscous flow, in particular, a viscous sublayer and the generation of eddies.

In the description of a viscous flow, the space scale equal to the relation of the viscosity coefficient to the velocity of the free stream $l_{m}$ plays an important role. Using it one reduces the diffusion equation to the dimensionless form depending only on one parameter, that is the dimensionless length of the plate, which is the Reynolds number.

The flow region, where the viscosity is manifested, is divided into three parts: the viscous sublayer, the body of the boundary layer and the mantle. The thick- 
ness of the viscous sublayer is $\sim 20 l_{m}$; that of the body of the boundary layer is $\sim 100 l_{m}$; the thickness of the mantle is more than $2000 l_{m}$. In the mantle, the diffusion momentum is close to zero, and it decreases near the body of the boundary layer, where the flow is nonpotential. The mantle size determines the boundary of the influence of the viscosity on the flow. The effects of the viscous flow-around cover the region which is ten times larger than the body of the boundary layer.

The body of the boundary layer is the region where the effect of the diffusion viscous momentum is significant.

The viscous sublayer is the region where the diffusion force producing the moment of force and generating the eddies is essential. The moment of force at the front edge of the plate is directed so that the eddy caused by it would press the flow to the plate surface. Moving along the plate the diffusion moment of force changes its orientation for the opposite one, which leads to the fact that the flow breaks off the plate surface, thus resulting in turbulence.

The eddy produced in the viscous sublayer has a small diameter and a high rotational velocity. Due to the diffusion momentum, the eddy increases in diameter and its angular velocity decreases. The eddy diameter also grows as the eddies combine. As a result, during the time, the flow passes the plate the eddy diameter increases up to the value of the order of the thickness of the boundary layer, with its angular velocity decreasing.

The momentum of the mantle has been obtained in the quadrature form, and it consists of the potential and nonpotential parts.

The consideration of the interaction of the gas with the surface of the plate that is flown around shows that the boundary condition of the adhesion of the flow to the plate surface is rough and it does not allow estimating the diffusion momentum near the front edge of the plate. The interaction of the flow with the plate is characterized by the friction coefficient. It determines the plate lengths for qualitatively similar flows as well as the value of the Reynolds number for the transition from the laminar flow to the turbulent one.

The momentum of the mantle has been obtained in the quadrature form, and it consists of the potential and nonpotential parts.

Here we obtain an analytical solution to the problem of viscous fluid flow around the plate. To understand a physical phenomenon, the analytical solution is more convenient, makes it easier to understand the essence of the phenomenon, and to identify the essential parameters that determine it. It greatly simplifies calculations for engineering applications in the development of structures that use fluid and gas flows, simplifies finding their optimal action, and so on.

Here, the theory of generalized functions [6] [7] was used to obtain an analytical solution. It allows you to get an analytical solution to a wide range of problems in mathematical physics that have real practical significance.

\section{Conflicts of Interest}

The author declares no conflicts of interest regarding the publication of this paper. 


\section{References}

[1] Ivanchin, A. (2018) Delusions in Theoretical Hydrodynamics. World Journal of Mechanics, 8, 771-831.

https://www.scirp.org/Journal/PaperInformation.aspx?PaperID $=87600$

[2] Loytsanskii, L.G. (2003) Fluid Mechanics. Drofa, Moscow.

[3] Landau, L. and Lifshits, E. (1987) Theoretical Physics: Fluid Mechanics. Pergamon Press, Oxford.

[4] Gallavotti, G. (2002) Foundations of Fluid Dynamics. Springer-Verlag, Berlin. https://doi.org/10.1007/978-3-662-04670-8

[5] Drazin, P.G. (2006) The Navier-Stokes Equations. A Classification of Flows and Exact Solutions. Cambridge University Press, Cambridge. https://doi.org/10.1017/CBO9780511526459

[6] Kanwal, R.P. (1983) Generalized Functions. Theory and Technique, Academic Press, Orlando.

[7] Vladimirov, V.S. (1971) Equation of Mathematical Physics. Marcel Dekker, New York.

[8] Landau, L. and Lifshits, E. (2005) Statistical Physics. Elsevier, London.

[9] Landau, L.D. and Lifshits, E.M. (1960) Mechanics. Pergamon Press Ltd., Oxford.

[10] Korn, G.A. and Korn, T.M. (2000) Mathematical Handbook for Scientists and Engineers. Dover Publications, New York.

[11] Riley, N. and Watson, E.J. (1993) Eccentric Oscillations of a Circular Cylinder in a Viscous Fluid. Mathematika, 40, 187-202. https://doi.org/10.1112/S0025579300006975

[12] Smith, S.H. (1991) The Rotation of Two Circular Cylinders in a Viscous Fluid. Mathematika, 38, 63-66. https://doi.org/10.1112/S0025579300006446 


\section{Appendix. Curl and Rotation}

Since this article is a continuation of [1], one more delusion in theoretical hydrodynamics should be noted. There exists a concept [2] that the value $\operatorname{rot} v / 2$ is the angular velocity of the rotation of the elementary volume. However, this is not so.

The concept of rotation implies a body of a finite size that cannot be determined for a point, since the rotation of a point is a meaningless concept. A curl is the differential operator meaningful for a point rather than for an extended object. Therefore, in a general case, it is wrong to say that a curl determines rotation.

It makes sense to determine rotation through a curl for solids whose curl of all points is the same. The linear velocity of an arbitrary point of a rotating solid in the cylindrical coordinates $r, \varphi, z$ with the $z$-coordinate as the axis of rotation is written as

$$
\boldsymbol{v}=\langle 0, \omega r, 0\rangle
$$

The broken brackets indicate that the vector is written in the cylindrical coordinate system. Hence, one derives

$$
\operatorname{rot} v=2 \omega\langle 0,0,1\rangle
$$

It is not right to compare rotv and the angular velocity for a fluid, which can be illustrated using two examples.

Plane - parallel flow. Let the flow velocity be given as

$$
\boldsymbol{v}=\{f(y), 0,0\}
$$

The above flow is nonpotential; it is impossible to produce it by a pressure difference; it can only be produced by viscosity. The flow moves forward, its motion is parallel to abscissa, there being a nonzero velocity curl at each point of the flow

$$
\operatorname{rot} v=\left\{0,0,-f^{\prime}(y)\right\} \not \equiv 0
$$

The vector (117) possesses one nonzero component, which is the $z$-coordinate, but there is no rotation. This movement cannot be called rotation as its trajectories are straight lines parallel to abscissa.

Eddy source. The field with the velocity

$$
\boldsymbol{v}=\frac{\omega}{x^{2}+y^{2}}\{-y, x, 0\}=\left\langle 0, \frac{\omega}{r}, 0\right\rangle
$$

is called an eddy source. In the cylindrical coordinates it has only the azimuth velocity rather than the zero one. The trajectories of fluid particles are concentric circles with the center at the origin of coordinates. The angular velocity of a fluid particle is $\omega / r^{2} \neq 0$, there exists rotation, however, $\operatorname{rot} v \equiv 0$.

These two examples show that the velocity curl and the angular velocity are different notions. 